New

Method

\title{
Increase Carotid Flow by Double Sheath Connection Technique to Reduce Cerebral Ischemia for Transcatheter Aortic Valve Implantation through Transcarotid Approach
}

\author{
Richard Tsai, MD, ${ }^{1}$ I-Ming Chen, MD, PhD,${ }^{1,2}$ Po-Lin Chen, MD,${ }^{1,2}$ Hsin-Bang Leu, MD, ${ }^{3}$ \\ Ying-Hwa Chen, MD, $\mathrm{PhD},{ }^{1,4}$ and Hsiao-Huang Chang, $\mathrm{MD}^{2}$
}

\begin{abstract}
Purpose: Transcarotid transcatheter aortic valve implantation (TAVI) is one alternative approach if unfavorable femoral access. However, this approach may cause cerebral vascular accidents (CVAs) by temporarily occluding common carotid artery (CCA). The purpose of this study is to develop a new method reducing cerebral ischemia during transcarotid TAVI. Methods: We inserted an 8- and 18-Fr. sheath in CCA with tip toward brain and aortic arch, respectively, and connected their side arms to create a bypass flow. Medtronic CoreValve was then delivered and deployed in position after pre-TAVI balloon dilatation.

Results: Three patients received this implantation. There were no CVAs or transient ischemic attacks (TIAs) after the procedure and all patients had been followed up uneventfully for 1 year. Conclusion: Our technique is feasible and potentially reduces stroke in transcarotid TAVI.
\end{abstract}

Keywords: transcatheter aortic valve implantation, transcarotid approach, stroke, sheath, carotid artery

\section{Introduction}

Transcatheter aortic valve implantation (TAVI) has developed as an alternative for aortic stenosis patients whose condition was intolerable undergoing conventional surgical aortic valve replacement (SAVR), and

${ }^{1}$ Department of Medicine, School of Medicine, National Yang Ming University, Taipei, Taiwan

${ }^{2}$ Division of Cardiovascular Surgery, Department of Surgery, Taipei Veterans General Hospital, Taipei, Taiwan

${ }^{3}$ Division of Healthcare and Management, Healthcare Center, Taipei Veterans General Hospital, Taipei, Taiwan

${ }^{4}$ Division of Cardiology, Department of Medicine, Taipei Veterans General Hospital, Taipei, Taiwan

Received: February 8, 2018; Accepted: March 1, 2018

Corresponding author: I-Ming Chen, MD, PhD. Department of Medicine, School of Medicine, National Yang Ming University, No. 201, Sec. 2, Shih-Pai Road, Taipei, Taiwan

Emails: alomar2099@gmail.com,imchen@vghtpe.gov.tw

(C)2018 The Editorial Committee of Annals of Thoracic and Cardiovascular Surgery. This work is licensed under a Creative Commons Attribution-NonCommercial-NonDerivatives International License. was reported to have similar or even better survival rate compared with SAVR.

Most of the TAVI was performed via transfemoral approach and other accesses, such as transaortic, transsubclavian, transapical, or transcarotid, would be considered a bail-out route if unfavorable femoral access. Mylotte et al. ${ }^{1)}$ reported a study of transcarotid TAVI without any cerebral vascular accidents (CVAs) but six cases $(6.3 \%)$ of transient ischemic attack (TIA) because the sheath for TAVI was placed in common carotid artery (CCA) and might temporarily decrease cerebral flow causing cerebral ischemia.

Hence, we developed a novel double sheath connection technique to decrease cerebral ischemia for transcarotid TAVI in the aim of reducing CVA.

In-vitro test of double sheath connection technique

Likittanasombut et al. ${ }^{2)}$ reported that the normal volume flow rate of non-stenotic CCA was around $337 \mathrm{~mL} /$ minute. To make sure how much flow we could offer by this double sheath connection technique, we performed 


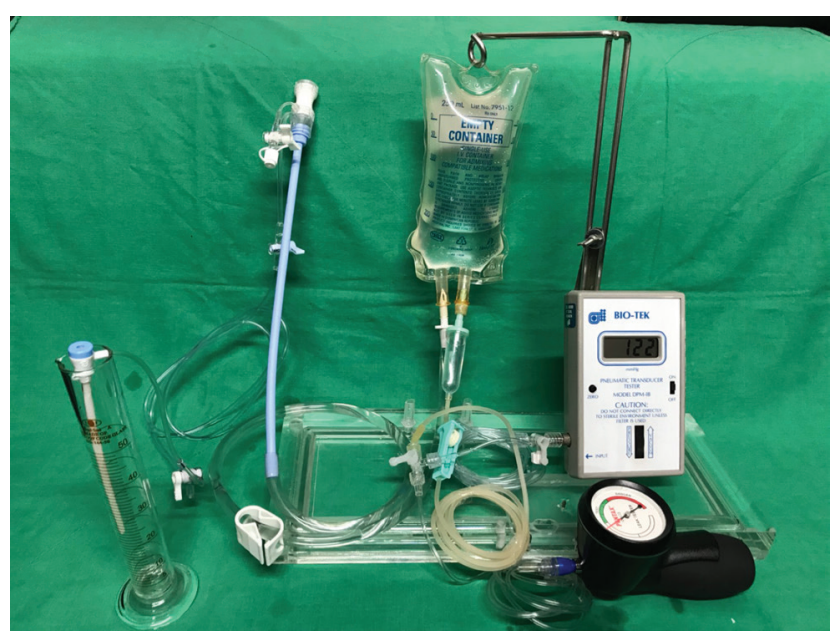

Fig. 1 An in-vitro test to confirm the actual flow by double sheath connection technique.

an in-vitro test (Fig. 1). We applied normal saline into the 18-Fr. sheath under the pressure around $120 \mathrm{mmHg}$ to see how much volume of normal saline could be obtained from the 8-Fr. sheath. We had tested 4 times and the flow was 195, 195, 175, and $202 \mathrm{~mL} /$ minute, respectively. The average flow from this in-vitro test was $191.75 \mathrm{~mL} / \mathrm{minute}$. The flow that we could offer by our technique is near $58 \%$ of the normal flow, which is believed to be better than almost no flow when an 18-Fr. sheath was inserted into carotid artery and occupied almost all the lumen of carotid artery.

\section{Technique description}

Under general anesthesia with brain oximeter monitor, patients are prepared in supine position with turning their head toward left to well expose right neck. An oblique incision was made along the medial side of sternocleidomastoid muscle to well expose right CCA. After giving heparin 8000 units or more to achieve activated clotting time (ACT) more than 300 seconds, we punctured the distal CCA and inserted an 8-Fr. by $5-\mathrm{cm}$ sheath by Seldinger maneuver under fluoroscopic guidance with sheath tip in external carotid artery to avoid possible internal carotid artery injuries. Another puncture was made just $1-2 \mathrm{~cm}$ below the previous 8 -Fr. sheath and an 18-Fr. sheath was inserted under stiff wire support and fluoroscopic guidance with sheath tip in ascending aorta (Fig. 2).

After adequate de-airing of both sheaths, we connected both side arms (Fig. 2) so that the blood flow from 18-Fr. sheath could be forward via the connected side arms to 8-Fr. sheath to perfuse brain (Fig. 3).

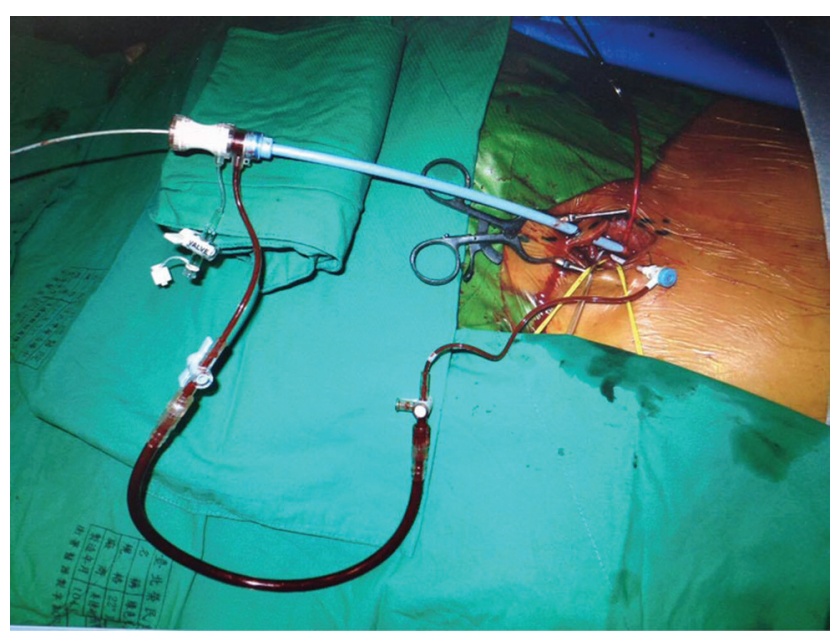

Fig. 2 After well position of both sheaths, the side arms were connected by extension tube with cautious de-airing.

We then cannulated aortic valve orifice from 18-Fr. sheath by straight guidewire. A pig-tail catheter was entered left ventricle through the wire and intra-ventricular pressure was measured after retrieval of the wire. Another stiff wire was inserted through the pig-tail catheter into left ventricle. Medtronic CoreValve was then delivered and accurately deployed in position after pre-TAVI balloon dilatation. After confirmation of final angiogram and retrieval of all the wires and sheaths in CCA, we clamped both ends of CCA and repaired the arteriotomy by 6-0 prolene sutures. The carotid angiography was also performed to check if there is any stenosis after sheath removal and carotid repair.

\section{Patients and Results}

From May 2013 to August 2017, there were 151 patients receiving TAVI in our institute. Three of them received transcarotid TAVI after preoperative computed tomography angiography of neck to confirm the quality of carotid artery. The institute review board has approved our study and informed consent was obtained. Patent 1 was an 83-year-old male with bilateral severe calcified femoral-iliac arteries and relatively small subclavian artery, which were not suitable for an 18-Fr. sheath. In addition, much mural thrombus and some ulcerative plaques were noted over abdominal aorta. So, we adopted transcarotid TAVI with $29 \mathrm{~mm}$ CoreValve. Patient 2 was an 87-year-old male with the history of chronic type B aortic dissection, which might cause aortic injury when advancing the CoreValve system through descending aorta. Therefore, we adopted transcarotid TAVI with 


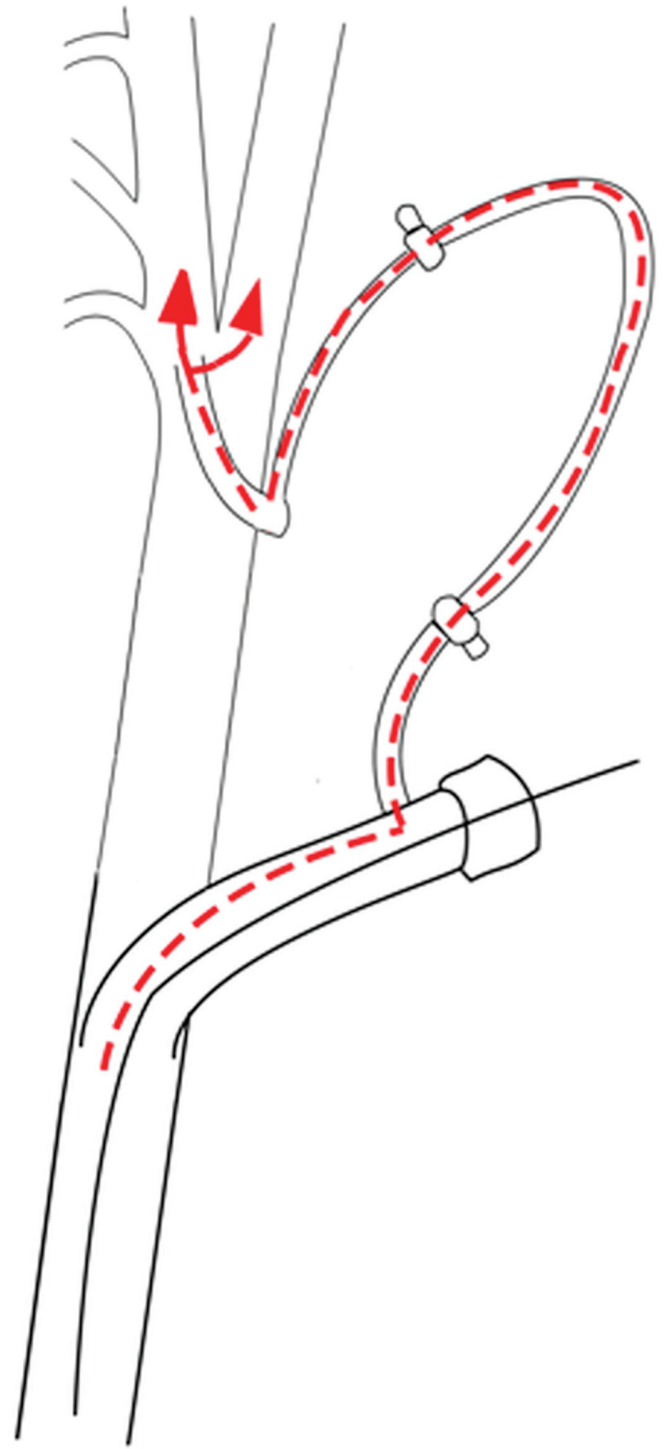

Fig. 3 The red dotted arrow depicted the blood flow from the big to small sheath toward the brain through the connected side arms.

$26 \mathrm{~mm}$ CoreValve. Patient 3 was an 81-year-old male with small subclavian and severe calcified femoraliliac arteries. So, we performed transcarotid TAVI with $29 \mathrm{~mm}$ CoreValve. The time from insertion of an 18-Fr. sheath to completion of CCA repair, which means the brain perfusion time by the connected sheath, was 32,42 , and 37 minutes in patients 1,2 , and 3 , respectively. The baseline brain oximetry was $75 \%, 62 \%$, and $66 \%$, and could be maintained no less than $72 \%, 64 \%$, and $64 \%$ during operation. There was neither CVAs nor TIAs after TAVI and all the patients followed up at our out-patient department uneventfully for 1 year.

\section{Discussion}

Transcarotid TAVI is much less common than transfemoral TAVI and should be considered one of the bail-out accesses when femoral artery is unavailable. Hypoperfusion causing CVAs is a concern in transcarotid TAVI. Inserting an 18-Fr. sheath $(6 \mathrm{~mm}$ in diameter) into CCA, especially in female, will almost block the carotid flow, since the mean diameter of a CCA is about $6.52 \mathrm{~mm}$ and $6.1 \mathrm{~mm}$ in Caucasians male and female. ${ }^{3)}$ Mathis et al. ${ }^{4)}$ reported that temporary balloon occlusion test of unilateral internal carotid artery within 15 minutes caused $1.6 \%$ CVAs. Generally, the transcarotid TAVI time, from insertion of an 18-Fr. sheath to removal, is at least 30 minutes, which probably cause higher incidence of CVAs. Hence, reducing possible CVA is mandatory for transcarotid TAVI.

Our design of double sheath connection technique is based on the similar concept to avoid distal leg ischemia in peripheral cannulation and establishing of cardiopulmonary bypass in the groin. We also performed in-vitro test to confirm the actual flow that this connected sheath could offer. To our knowledge, our design is the first to demonstrate in carotid artery perfusion. Under the support of our technique, much more invasive transapical or direct aorta approach for TAVI could be avoided.

The disadvantage of our technique is possible air or thromboembolism into brain and possible distal carotid artery injury when placing an 8-Fr. sheath. First, it is always important to maintain ACT above 300 seconds and de-air meticulously. Second, the 8-Fr. sheath indwelling should be performed cautiously under fluoroscopic guidance and contrast angiography to confirm the adequate position of sheath without any carotid artery injuries. Third, the offered flow is only 50\%-60\% of normal flow. However, it is better than nothing since an 18-Fr. sheath would block almost all the flow of carotid artery.

A temporary femoral-to-carotid shunt is one of the approaches to reduce CVAs. ${ }^{5)} \mathrm{A}$ sheath was inserted into the ipsilateral femoral artery and connected to a CCA shunt, resulting an antegrade perfusion to brain. However, our technique is easier without establishment of another femoral access. A subclavian-to-CCA bypass is another option for reducing CVAs. $\left.{ }^{6}\right)$ However, additional subclavian anastomosis was required.

There are some limitations in our study. First, we did not include a control group for comparison. Next, the 
sample size was small. However, perioperative brain oximetry was monitored to ensure brain perfusion. And the current results suggested that our technique is a feasible bail-out procedure and is not inferior to the femoroto-carotid shunt ${ }^{5)}$ or subclavian-to-carotid bypass. ${ }^{6}$ )

\section{Conclusion}

Our technique for transcarotid TAVI potentially reduces hypoperfused CVAs and is a feasible bail-out alternative if transfemoral TAVI is unfavorable. Further studies are necessary to confirm the efficacy.

\section{Acknowledgments}

The authors would like to thank for the help from Taiwan Association of CardioVascular Research.

\section{Disclosure Statement}

None.

\section{References}

1) Mylotte D, Sudre A, Teiger E, et al. Transcarotid transcatheter aortic valve replacement: feasibility and safety. JACC Cardiovasc Interv 2016; 9: 472-80.

2) Likittanasombut $P$, Reynolds $P$, Meads $D$, et al. Volume flow rate of common carotid artery measured by Doppler method and Color Velocity Imaging Quantification (CVI-Q). J Neuroimaging 2006; 16: 34-8.

3) Krejza J, Arkuszewski M, Kasner SE, et al. Carotid artery diameter in men and women and the relation to body and neck size. Stroke 2006; 37: 1103-5.

4) Mathis JM, Barr JD, Jungreis CA, et al. Temporary balloon test occlusion of the internal carotid artery: experience in 500 cases. AJNR Am J Neuroradiol 1995; 16: 749-54.

5) Azmoun A, Amabile N, Ramadan R, et al. Transcatheter aortic valve implantation through carotid artery access under local anaesthesia. Eur J Cardiothorac Surg 2014; 46: 693-8; discussion 698.

6) Magalhães MA, Souza JM, Grube E. Transcatheter aortic valve implantation through a diseased left common carotid artery: combined approach with endarterectomy and left carotid-subclavian bypass. Catheter Cardiovasc Interv 2013; 81: 618-22. 